\title{
Biological control of bloody diarrhea by using recombinant bacterial ghost system
}

\author{
${ }^{1}$ Shayma`a Jamal Ahmed ${ }^{2}$ Nawfal K.Yas \\ ${ }^{1,2}$ Anatomy department, College of Medicine, Baghdad University.
}

\begin{abstract}
Diarrhea is the most common illness affecting human. It is defined as passing three or more unformed stools in 24 hours. The most common causes of diarrhea include the following: Bacterial infections, viral infections, parasites, functional bowel disorders, intestinal diseases, food intolerance, sensitivities and reaction to medicines. People who visit certain foreign countries are at risk for traveler's diarrhea (TD).

Bacterial ghosts are empty cells with intact envelopes, they have a natural outer surface make-up which provides them with the original targeting functions of the pathogen they are derived from and are thus able to induce strong local immunity, ghost are produced by controlled expression of cloned bactriophage phi x 174 lysis gene.

We have evaluated a new strain of Salmonella typhi Ty2la (GEB135) which has two plasmids CFA/I $(60000 \mathrm{kbp})$ and pASH (65000kbp) to use as a new approaches in biological controls of pathogenic bacteria and it has extended for recombinant bacterial ghost delivery system.
\end{abstract}

Key words: Diarrhea, ghost delivery system, Salmonella typhi Ty2la.

\section{Introduction}

Having diarrhea means passing loose stools three or more times a day. Acute diarrhea is a common problem that usually lasts 1 or 2 days and goes away on its own, acute diarrhea is usually caused by a bacterial, viral, or parasitic infection. Chronic diarrhea is usually related to a functional disorder such as irritable bowel syndrome or an intestinal disease such as Crohn's disease (Dupont, 1997).

Shigella toxin (stx) producing Escherichia coli (STEC) is among the most common causes of foodborne diseases. This organism is responsible for several human gastro intestinal illnesses, including non bloody or bloody diarrhea. Especially in children, these diseases may be affected by neurologic and renal complications, including hemolytic uremic syndrome (HUS) (Ramaswamy\& Jacobson, 2001).

Most outbreaks and sporadic cases of bloody diarrhea and HUS have been attributed to strains of STEC serotype O157:H7. However, in Europe and recently in the United States, the role of non-O157 STEC strains (e.g., O26:H11/H-, O91:H21/H-,O103:H2, O111:H-, O113:H21, O121:H19, O128:H2/H-, and O145:H28/H-) as causes of HUS, bloody diarrhea, and other gastrointestinal illnesses is being increasingly recognized (Kappeli et al., 2011).

In the recombinant bacterial ghost system, foreign proteins are attached on the inside of the inner membrane as fusions with specific anchor sequence, ghosts have a sealed periplasmic space and the export of proteins into this space vastly extends the capacity of ghost or recombinant ghost to function as carriers of foreign antigens. In addition S-layer proteins forming shell-like self assembly structures can be expressed in candidate vaccine strains prior to E-mediated lysis. Such recombinant S-layer proteins carrying foreign epitopes further extend the possibilities of ghost as carriers of foreign epitopes (this make the ghosts have inherent adjuvant properties)(Lubitz et al., 1999 ).

In our view, the extended recombinant bacterial ghost system represents new approaches in vaccine development and biological controls to provide alternative platform technologies which can create better qualities of combination vaccines. These candidate vaccines can be adherence to small intestine by CFA/I factor and can combine to the CFA pathogenic bacteria and lysis by E-mediated lysis system.

\subsection{Bacterial strain \& plasmids}

\section{Research Methods}

- In this study the strain used is Salmonella typhi Ty21a (GEB130) (Ahmed,1997) which has the phenotype \& genotype: $\mathrm{GalE}^{-}$(galactose mutant), aro- (aromatic amino acid mutation), CFA/I (a plasmid with encode colonization factor antigen type I (60000kbp) ) and Rif® , Cm® (resistance to the antibiotics: Rif®: Rifampicin $(50 \mu \mathrm{g} / \mathrm{ml}), \mathrm{Cm} \circledast$ : Chloramphenicol $(50 \mu \mathrm{g} / \mathrm{ml})$ ).- pASH: it is $65000 \mathrm{kbp}$, resistance to Tc® (resistance to Tetracycline antibiotic $(10 \mu \mathrm{g} / \mathrm{ml})$ ) and point mutation in the operator of $\lambda$ ( $\lambda$ pr mut ) (Ahmed, 1997). The bacterial strain was maintained on Brain Heart Infusion Agar (Oxford) with selection marks.

- Colonization, factor assay and isolation: Bacteria was grown on CFA agar and tested for Mannose Resistance Hemagglutiation with human Blood group A (Ahmed,1997). 


\subsection{Transformation \& DNA isolation}

pASH was transformed to Salmonella typhi Ty21a (GEB130) and got Salmonella typhi Ty21a (GEB135) strain according to standard procedures and the DNA was isolated by alkaline lysis (Sambrook,et al. 1989).

\subsection{Growth \& lysis of bacterial culture}

Salmonella typhi Ty21a (GEB135) (the transformed strain with pASH plasmid) was grown on Luria broth (Witte, et al. 1992) and was incubated at room temperature for 2 days. A mutant clone was selected for capability to grow at $37 \mathrm{C}^{\circ}$ and to lyse at $42 \mathrm{C}^{\circ}$. Growth and lysis of bacterial cultures was monitored by measuring the optical density at $600 \mathrm{~nm}(\mathrm{OD}=600)$, at $37 \mathrm{C}^{\circ}$ bacteria was able to grow with a normal doubling rate, while at $42 \mathrm{C}^{\circ}$ bacterial lysis was detected for $15 \mathrm{~min}$ after temperature shift-up (Jechlingek, et al. 1999 ).

\subsection{Experimental bacterial lysis}

In this test we used enterotoxigenic E.coli (ETEC) strain (isolated from patient with bloody diarrhea from teaching laboratory in the Medical City Hospital). The cultures (Salmonella typhi Ty21a (GEB135) \& E.coli (ETEC)) were grown in Luria broth and then mixed $1 \mathrm{ml}$ of each one for 2 hours with incubation at $37 \mathrm{C}^{\circ}$ without movement, finally bacteria were cultured in transconjugant media (O`cennell,1984) and repeat the step (2.3) of growth \& lysis of bacterial culture.

\subsection{DNA extraction}

DNA was extracted from Salmonella typhi Ty21a (GEB130), Salmonella typhi Ty21a (GEB135) and E.coli (ETEC) then DNA concentration and purity were estimated according to Sambrook (Sambrook, et al. 1989) by using ultra violated spectrophotometer (CECIL,CE7200), finally the DNA was electrophoresed by using $1 \%$ agrose gel electrophoresis at $3 \mathrm{~V} / \mathrm{cm}$ for $90 \mathrm{~min}$.

\section{Results}

Salmonella typhi Ty21a (GEB135) was grown and lysed by measuring the optical density at $600 \mathrm{~nm}$ $\left(\mathrm{OD} 600=0.47\right.$ ) at $37 \mathrm{C}^{\circ}$ (bacteria was able to grow) and after temperature shift up at $42 \mathrm{C}^{\circ}$ for 15 min the OD $600=0.27$ (bacteria was lysed). This bacterial strain has two plasmids the first one is CFA/I and the second is pSH (fig-1).

The Transconjugant bacteria from Salmonella typhi Ty21a (GEB135) and E.coli (ETEC) were also grown and lysed by measuring the optical density at $600 \mathrm{~nm}(\mathrm{OD} 600=0.3)$ at $37 \mathrm{C}^{\circ}$ and after temperature shift up at $42 \mathrm{C}^{\circ}$ for $15 \mathrm{~min}$ the OD $600=0.12$ fig- 2 .

Fig-3 shows the shape of the bacteria after temperature shift up where bacterial cell becomes empty and disrupted.

\section{Discussion}

In this study we used Salmonella typhi Ty21a (GEB135) as a delivery system for recombinant bacterial ghost system, this strain has the capacity to protect against the typhoid fever. The strain was found to be safe and effective in field trials, it can serve as a useful carrier for immunogenic determinants such as I antigen from Shiglla sonnei which makes it protective against shigllosis and typhoid fever, hence, it can also be used to express an enterotoxigenic E.coli (Ahmed, et al. 2001).

After transformation, Salmonella typhi Ty21a (GEB135) has two plasmids (fig-1) the first one is CFA/I (6000kbp) \& pASH (65000kbp)) with resistance to Rif® , Cm® \& Tc ${ }^{\circledR}$ (selective markers), this bacteria was used as a delivery system to enterotoxigenic E.coli after it has been transconjugated experimentally and the cells were lysed after temperature shift up which were empty and distrupted (fig-2). Cell lysis of gram-negative bacteria can be efficiently achieved by expression of the cloned lysis gene $\mathrm{E}$ of bacteriophage phix 174, gene $\mathrm{E}$ expression is tightly controlled by the rightward $\lambda \mathrm{pR}$ promoter and the temperature-sensitive repressor cI857 on lysis plasmid the resulting empty bacterial cell envelopes, called bacterial ghosts (Jenchlinggel, et al. 1999)

The empty bacterial cell envelopes (bacterial ghosts) raised from pathogenic gram-negative bacteria are under investigation as vaccine candidates and carriers of forgein antigens (Szostak ,et al. 1996).

The production of ghost by protein E-mediated lysis is based on the tightly controlled expression of the cloned lysis gene E of bacteriophage phi x 174 lysis gene. The gene E encodes a hydrophobic polypeptide of 91 amino acids that forms a transmembrane tunnel in the bacterial cell wall through which the cytoplasmic contents are expelled (SchÖn, et al. 1995).

The resulting bacterial ghosts share functional and antigenic determinants of the envelope with their living counterparts, as the protein E does not cause physical or chemical denaturation processes to bacterial surface structure (Witte, et al. 1992). In various lysis plasmids gene E expression, which is lethal to the host cell, is controlled by the rightward phage $\lambda \mathrm{pR}$ promoter and the corresponding temperature-sensitive repressor, cI857, 
which is inactivated at temperatures higher than $30 \mathrm{C}^{\circ}$ (Remaut, et al. 1981). Bacterial lysis due to expression of gene $\mathrm{E}$ is induced by temperature shift of the growing culture from $28 \mathrm{C}^{\circ}$ to $42 \mathrm{C}^{\circ}$, in E.coli growth of the culture stops approximately 10 to $15 \mathrm{~min}$ after the induction of gene E expression. Since pathogenic bacteria infecting humans or animals mostly grow at an ambient temperature of $37 \mathrm{C}^{\circ}$ or more, it is favorable to cultivate these bacteria at their natural ambient growth temperature, another advantage of cultivating bacteria at temperatures higher than $28 \mathrm{C}^{\circ}$ is the faster growth rate and higher cell mass production (Jenchlinggel,et al. 1999) .

\section{Refernce}

[1] Ahmed SH.J.(1997). The transfer of genetic and immune-determinant to, and genetic improvement of Salmonella typhi strain used as oral vaccine.(M.Sc. thesis). College of Science. Baghdad Unversity.

[2] Ahmed SH.J.,Al-zaag A.A.\& Ali N.A.(2001). A modified Salmonella typhi strain suitable as oral vaccine against Escherichia coli and typhoid infections. J. Fac. Med. 43(1) 77-80.

[3] DuPont H.L.(1997).Practice parameters committee of American college of Gastroenterology.Guidelines on acute infectious diarrhea in adults. The American J. of Gastroenterology. 92 (11). 1962-75.

[4] Jechlinger W.,Szostak M.P., Witte, A.\& Lubitz, W.(1999). Altered temperature induction sensitivity of the Lambda pR/CI857 system for controlled gene E. in expression in Escherichia coli . FEMS Microbiology letters. 173. 347-52.

[5] Käppeli U.,Hächler H., Giezendanner N., Beutin L.\& Stephan R.(2011). Human Infections with Non-o157 Shiga Toxin-producing Escherichia coli , Switerland, 2000-2009. Emerging Infectious Diseases.17(2).180-85.

[6] Lubitz W.A., Witte F., Eko M., Kamal W., Jechlinger E.,Brand J., Marchart W., Haidinger V., Huter D., Felnerova N. et al. (1999). Extended recombinant bacterial ghost System.J. Biotechnol. 73. 261-73.

[7] Oconnell M.P.(1984). Genetic transfer in prokaryotes: Transformation, Transduction and conjugation.pp.2-13. In Puhler A.\& Timmis K.N.(eds.). Advanced Molecular Genetics. Springer Verlag. Berlin.

[8] Ramaswamy K., Jacobson K.(2001). Infectious diarrhea in children. Gastroenterology clinics of North America.30(3).611-

[9] Sambrook J., Fritsoh E.F \& Manaitis T.( 1989) Molecular cloning: A laboratory manual $2^{\text {nd }}$ edition.

[10] SchÖn P., Schrot G., Wanner G., Lubitz w.\& Witte A.(1995). Two-stage model for integration of the lysis protein E of bacteriophage phiX174 into cell envelope of E.coli .FEMS Microbiol. Rev.17.207-12.

[11] Szostak M., Hensel A.,Eko F.O., Klein R., Auer T., Mader H.,Haslberger A.,Bunka S.,Wanner G.\& Lubitz w.(1996). Bacterial ghosts: non living candidate vaccines. J.Biotechnol. 44.161-70.

[12] Witte A., Wanner G.\& Lubitz W.(1992). Dynamics of phiX174 protein E-mediated lysis of Escherichia coli . FEMS Microbiology Rev.17. 207-12.

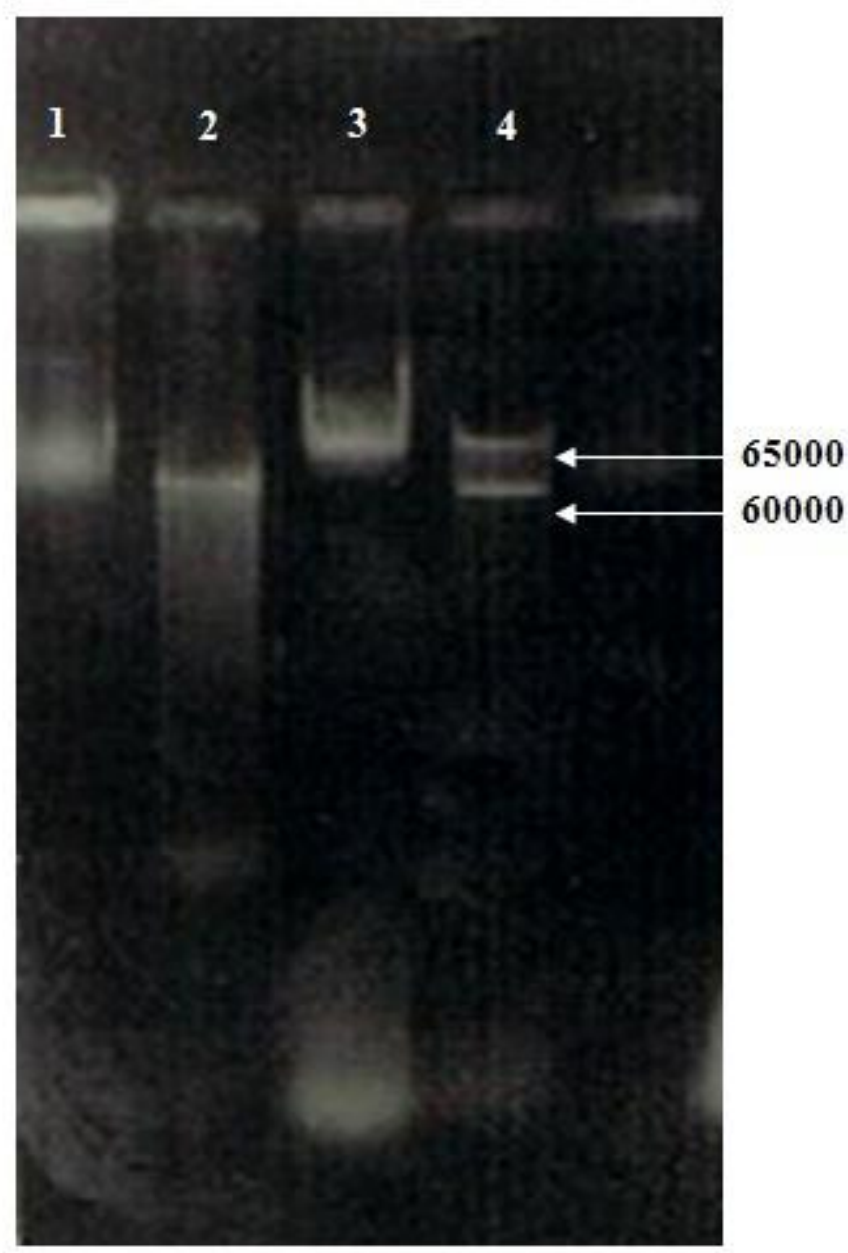




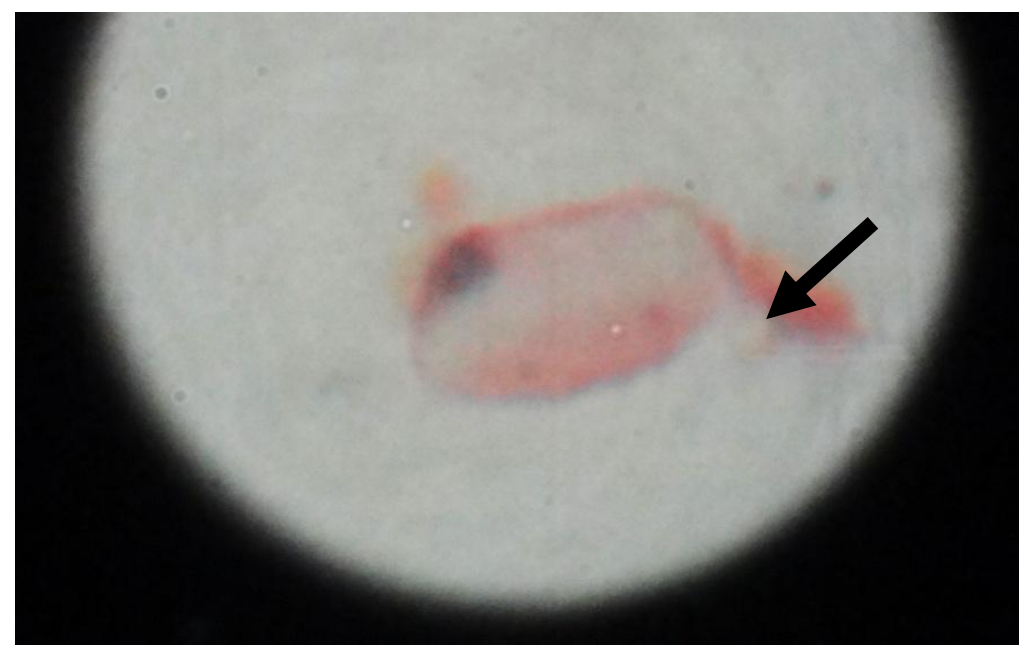

Fig-2- E.coli after it has been transconjugated experimentally and the cells were lysed after temperature shift up which were empty and disturbed.

Figure-3: E.coli (ETEC) after temperature shift up (42c $\square$ ), an arrow indicates the lysis of bacteria.

Figure-1:Lane 1,2 Salmonella typhi Ty21a (GEB130) with CFA/I plasmid (60000bp), Lane 3 pASH plasmid(65000bp), Salmonella typhi Ty21a (GEB135) with 2 plasmids pASH\& CFA/I. Electrophoresis was carried out in $1 \% \mathrm{CFA} / \mathrm{I}$ agrose gel supplied with ethidium bromide at $(3 \mathrm{~V} / \mathrm{cm})$ for 90 mints. 